American J. of Engineering and Applied Sciences 3 (1): 64-67, 2010

ISSN 1941-7020

(C) 2010 Science Publications

\title{
Electrical and Optical Properties of N, N`-Bis (Inaphthyl)-N,N`-Diphenyl-1,1'-Biphenyl- 4,4-Diamine as Hole Transport Layer in Organic Light Emitting Devices
}

\author{
${ }^{1}$ M.Y. Lim, ${ }^{1}$ W.M.M. Yunus, ${ }^{1}$ Z.A. Talib, ${ }^{2}$ A. Kassim, ${ }^{3}$ C.F. Dee and ${ }^{3}$ A. Ismail \\ ${ }^{1}$ Department of Physics, Faculty of Science, \\ University Putra Malaysia, 43400 UPM, Serdang, Selangor, Malaysia \\ ${ }^{2}$ Department of Chemistry, Faculty of Science, \\ University Putra Malaysia, 43400 UPM, Serdang, Selangor, Malaysia \\ ${ }^{3}$ Institute of Microengineering and Nanoelectronics, \\ University Kebangsaan Malaysia, 43600 UKM Bangi, Selangor, Malaysia
}

\begin{abstract}
Problem statement: The aim of this research was to study the electrical and optical properties of N, N`-bis(Inaphthyl)-N,N`-diphenyl-1,1`-biphenyl-4,4`-diamine (NPB) organic materials often used as hole transport layer in Organic Light-Emitting Devices (OLED). Approach: The NPB layer was prepared using the thermal evaporation method. From photoluminescence spectra, two peaks at 630 and $480 \mathrm{~nm}$ were observed with $55 \mathrm{~nm}$ NPB. The electrical conductivity was strongly influenced by the layer thickness. Results: The energy band gap of each NPB layer was successfully presented in the range of 2.4-2.9 eV. Conclusion: This study successfully showed the effect of different thickness of NPB in OLED.
\end{abstract}

Key words: Thermal evaporation, photoluminescence, energy band gap, electrical conductivity

\section{INTRODUCTION}

Various organic light emitting diode displays have been investigated in recent years. Despite their excellent performance, various techniques have been recently reported to further improve their efficiency. The basic structure of an OLED is shown in Fig. 1 and typically consists of three organic semiconductor layers sandwiched between two electrodes. The electron-injecting electrode consists of a low work function metal alloy, deposited by vacuum evaporation. Vacuum evaporation is widely to use for preparation OLEDs. OLEDs structure consists of two important layer which is hole tranposrt layer (HTL) and electron transport layer (ETL) connected by two electrodes. Indium Tin Oxide (ITO) as an anode on the bottom, hole-injecting, electrode (Paul et al., 1997). Upon recombination, energy is released as light, which is emitted from the light-transmissive anode and substrate.

Currently, there is great interest in the study of OLEDs containing small molecules as emitting layer. In addition to applications in OLEDs, these molecules have been found to be useful in applications such as optical devices, luminescence probes in biomedical assays, luminescence sensors for chemical species and fluorescent lighting (Kido and Okamoto, 2002; Silva and Pereira, 2006).

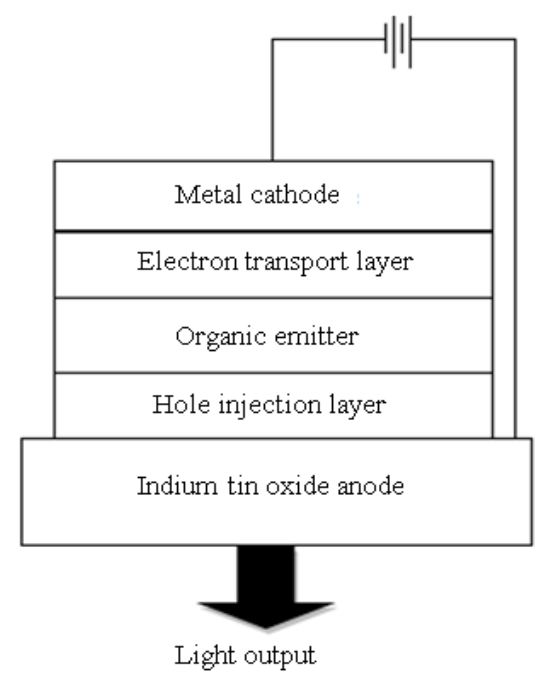

Fig. 1: The basic structure for organic light emitting devices

Corresponding Author: W.M.M. Yunus, Department of Physics, Faculty of Science, University Putra Malaysia, 43400 UPM, Serdang, Selangor, Malaysia Tel: + (603)89466684 Fax: + (603)89454454 


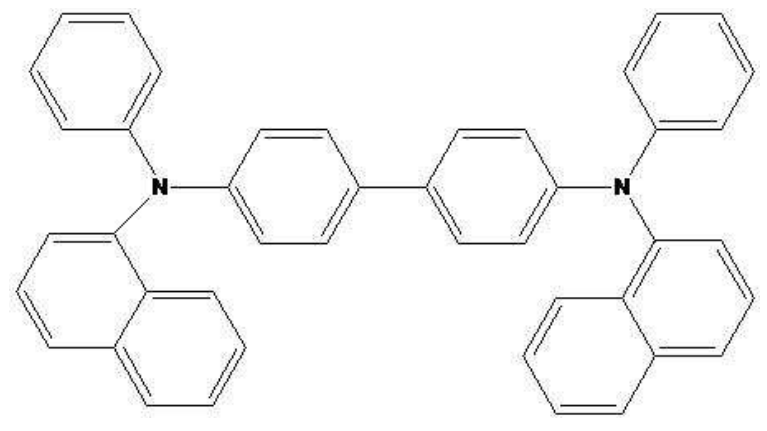

Fig. 2: Molecular structure of N,N`-bis(Inaphthyl)-N, $\mathrm{N}$-diphe.nyl-1,1`-biphenyl-4,4`-diamine

OLED development needs to include optimization of device structure, development of novel materials and improvement of manufacturing techniques and cost. Electrical doping of charge transport layers of organic light emitting diodes is one step towards this direction. One of the most widely used Hole Transport Layer (HTL) in OLED is N,N'-bis(Inaphthyl)-N,N'diphenyl-1,1'-biphenyl-4,4'diamine(NPB) (Fig. 2). This is because NPB can be manufactured readily and is thus abundantly available even though its $\mathrm{Tg}$ at $98^{\circ} \mathrm{C}$ is a trifle low which may affect its morphological stability at high operating temperatures (Hunga and Chen, 2002).

In the present study, different thickness NPB layer was prepared by vacuum evaporation method. The effect of thickness of NPB on the energy band gap, electric conductivity and photoluminescence of the NPB was investigated.

\section{MATERIALS AND METHODS}

Experimental: For the preparation of NPB organic layer, Indium Tin Oxide (ITO) was cut into square plates $(0.02 \times 0.020 \mathrm{~m})$ glass and used as substrate. The ITO glasses were immersed in ultrasonic baths with acetone for $10 \mathrm{~min}$. Then, the ITO glasses were rinsed in deionized water for $10 \mathrm{~min}$ and then blow dry with nitrogen gas. This procedure was used to remove organic contamination and particles from the ITO surface. Different thickness of organic layers were deposited on the ITO at a rate of $2.5 \mathrm{~A} / \mathrm{s}$ from resistively heated tungsten boats at a base pressure of $1.0 \times 10^{-5}$ Torr.

Current-voltage measurements were made with a Keithley source meter (model 2400). The thickness of the layers was measured by Tencor P-12 disk profile. The PL of the film was measured by a EL spectra USB 2000 FLG Spectrofluorometer.

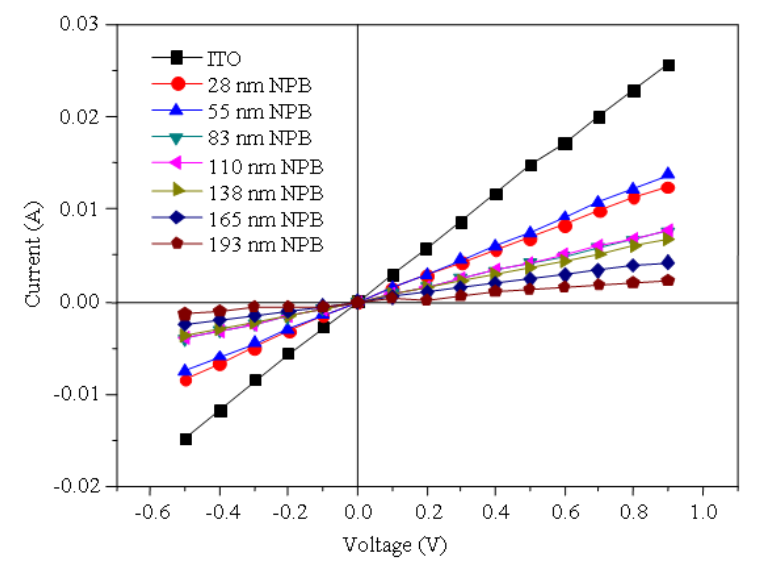

Fig. 3: Current-voltage curve for all different thickness of NPB

\section{RESULTS}

Figure 3 shows the current versus voltage curve of the seven NPB samples with different thickness. The electrical resistance $(\mathrm{R})$ of a circuit component or device is defined as the ratio of the voltage applied (V) to the electric current (I) which flows through it. Observation of Fig. 3 shows that the resistance decrease as the thickness of the NPB layer increased.

The spectra of optical absorption measurements were made over the wavelength range of 360-800 $\mathrm{nm}$. The variation in the absorbance with wavelength is shown in Fig. 4 for NPB with varying thickness. The spectra observed are similar. The expected variation of the absorption coefficient $(\alpha)$ with photon energy is given by:

$(\alpha h v)^{2}=C\left(h v-E_{g}\right)$

Where:

$\mathrm{c}=\mathrm{A}$ constant

$\mathrm{E}_{\mathrm{g}}=$ The optical band gap

hv $=$ Photon energy

The plot of $(\alpha h v)^{2}$ as a function of hv follows a straight line (fundamental absorption). Hence, in 2.4$2.9 \mathrm{eV}$ range, the absorption spectrum can be described by Eq. 1. The energy axis intercept of the least-square fit to the linear part yields the optical band gap $\mathrm{E}_{\mathrm{g}}$.

The room-temperature absorption spectrum for different thickness of NPB is shown in Fig. 6. In order to further optimize device structure so as to achieve optimal PL performances, the thickness of NPB host layer was first changed 
Am. J. Engg. \& Applied Sci., 3 (1): 64-67, 2010

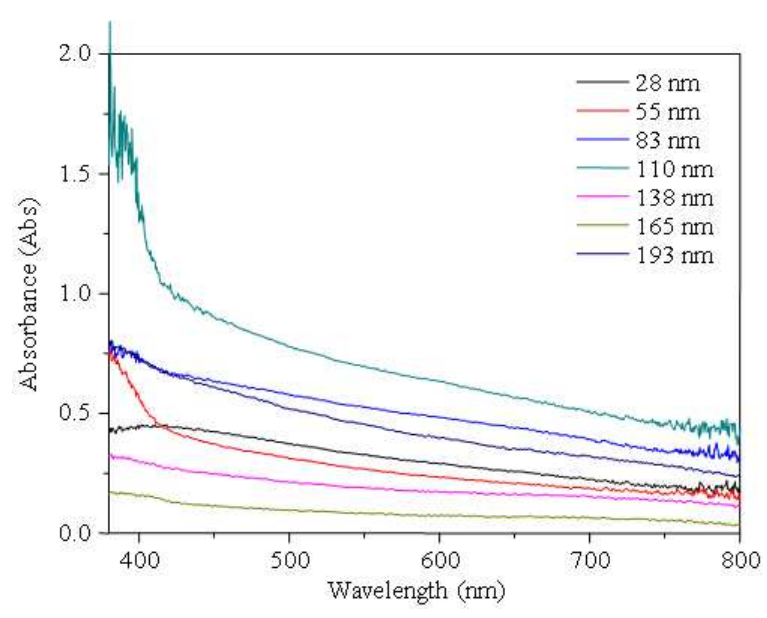

Fig.4: The absorbance versus wavelength for different thickness of NPB

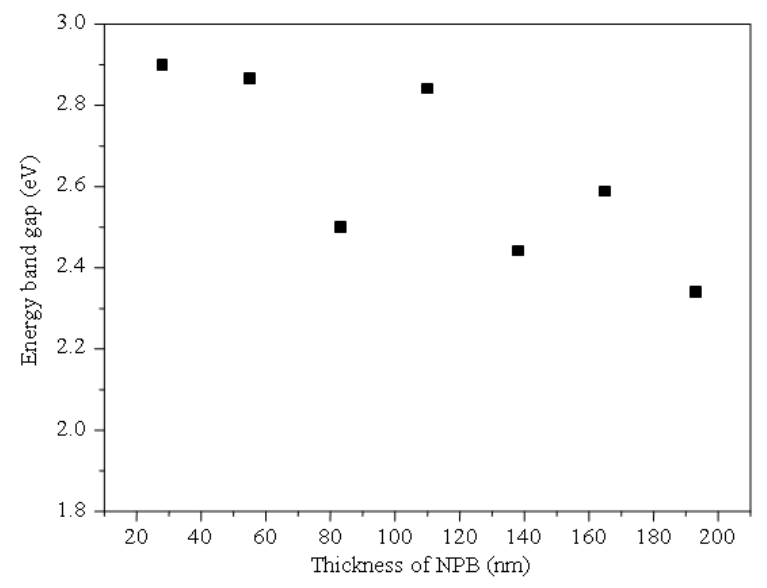

Fig. 5: Energy band gap for different thickness of NPB

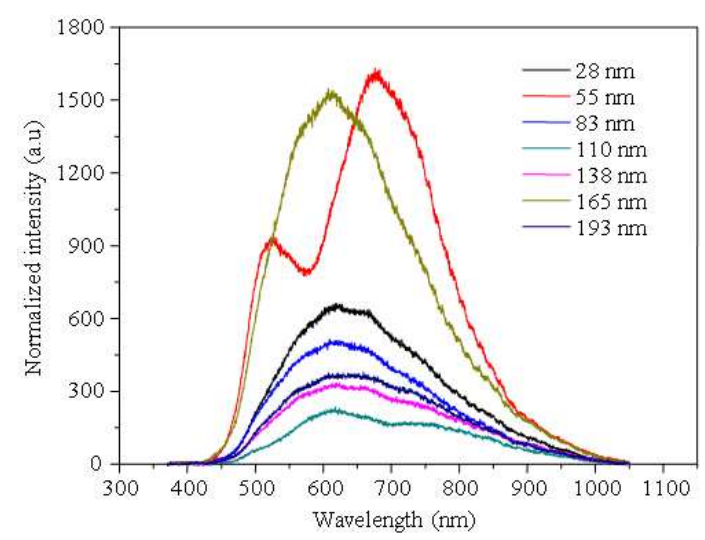

Fig. 6: The PL performance with the different thickness of NPB

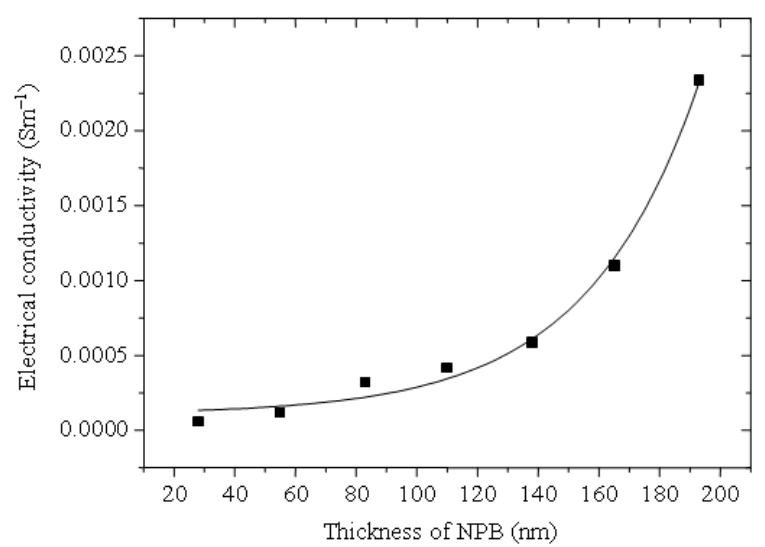

Fig. 7: Electrical conductivity for all different thickness of NPB

\section{DISCUSSION}

The electrical behavior of single layer OLEDs is known to have a strong linked to the NPB due to its mobility (Fig. 7). The electrical conduction is controlled by the energy states in the semiconductor bulk that usually arise from localized defects (Wolfgang et al., 2001).

From the result, the $\mathrm{E}_{\mathrm{g}}$ decrease with the increase thickness of NPB (Fig. 5). These results can be easily correlated with the efficient hole transport inside the HTLs as the analysis of energy (Zhi-Feng et al., 2003). It is also can expected that the improvement is on account of a good balance between the injected electrons and holes in the OLED devices.

The thickness of the NPB host strongly affected the PL performances and as it was thinned to be $55 \mathrm{~nm}$, the devices exhibited a peak efficiency and a better color coordinates, i.e., color saturation. It can be seen that the PL spectra of the $55 \mathrm{~nm}$ NPB have two peak located at 480 and $630 \mathrm{~nm}$. This indicated that the $55 \mathrm{~nm}$ NPB has intermolecular interaction between ITO stronger than other thickness of NPB (Lingling et al., 2005; Avendano et al., 1999).

\section{CONCLUSION}

For the PL spectra, two new peaks at 630 and $480 \mathrm{~nm}$ were observed for $55 \mathrm{~nm}$ NPB for the white light. The electrical conductivity was strongly dependence on the layer thickness. The $\mathrm{E}_{\mathrm{g}}$ decrease with the increase thickness of NPB. Thus, a correlation between the $\mathrm{E}_{\mathrm{g}}$ and electrical conductivity in NPB has been investigated. 


\section{REFERENCES}

Avendano, F.M., E.W. Forsythe and G. Yongli, 1999. The growth modes of NPB on indium tin oxide. Synthet. Metals, 102: 910-911. DOI: 10.1016/S0379-6779(98)00951-5

Hunga, L.S. and C.H. Chen, 2002. Recent progress of molecular organic electroluminescent materials and devices. Mater. Sci. Eng., R39: 143-222. http://huniv.hongik.ac.kr/ ekim/Ref_OS/242-4.pdf

Kido, J. and Y. Okamoto, 2002. Organo lanthanide metal complexes for electroluminescent materials. Chem. Rev., 102: 2357-2368. DOI: 10.1021/cr010448y

Lingling, R., K. Shi-Zhao, Z. Hui-Min, W. Li-Jun, P. Ge-Bo, Z. Chuan-Feng and B. Chun-Li, 2005. Investigation of ITO surface modified by NPB and arachidic acid LB films. Colloids and Surfaces A: Physicochem. Eng. Aspects, 257-258: 433-437. DOI: 10.1016/J.COLSURFA.2004.10.097

Paul, E.B., R.F. Stephen and M.E. Thompson, 1997. Prospects and applications for organic lightemitting devices. Curr. Opin. Solid State Mater. Sci., 2: 236-243. DOI: $10.1016 / \mathrm{S} 1359-$ 0286(97)80072-1
Silva, V.M. and L. Pereira, 2006. The nature of the electrical conduction and light emitting efficiency in organic semiconductors layers: The case of [mMTDATA]-[NPB]-Alq3 OLED. J. Non-Crystalline Solids, 352: 5429-5436. $\quad$ DOI: 10.1016/J.JNONCRYSOL.2006.08.016

Wolfgang, B., S. Berleb and A.G. Muckl, 2001. Device physic of organic light emitting diode based on molecular material. Organ. Elect., 2: 1-36. DOI: 10.1016/S1566-1199(01)00009-X

Zhi-Feng, Z., D. Zhen-Bo, L. Chun-Jun, Z. Meng-Xin and X. Deng-Hui, 2003. Organic light-emitting diodes with a nanostructured $\mathrm{TiO}_{2}$ layer at the interface between ITO and NPB layers. Displays, 24: 231-234. DOI: 10.1016/J.DISPLA.2004.01.010 